\title{
Inhibition of the unfolded protein response reduces arrhythmia risk after myocardial infarction
}

\author{
Man Liu, ${ }^{1}$ Hong Liu, ${ }^{1}$ Preethy Parthiban, ${ }^{2}$ Gyeoung-Jin Kang, ${ }^{1}$ Guangbin Shi, ${ }^{3}$ Feng Feng, ${ }^{1}$ Anyu Zhou, ${ }^{3}$ Lianzhi Gu, ${ }^{1}$ \\ Courtney Karnopp, ${ }^{2}$ Elena G. Tolkacheva, ${ }^{2}$ and Samuel C. Dudley Jr. ${ }^{1}$
}

'Division of Cardiology, Department of Medicine, the Lillehei Heart Institute and 'Department of Biomedical Engineering, University of Minnesota, Minneapolis, Minnesota, USA. ${ }^{3}$ Division of Cardiology, Department of Medicine, the Warren Alpert School of Medicine, Brown University and Lifespan Cardiovascular Research Center, Providence, Rhode Island, USA.

\begin{abstract}
Ischemic cardiomyopathy is associated with an increased risk of sudden death, activation of the unfolded protein response (UPR), and reductions in multiple cardiac ion channels. When activated, the protein kinase-like ER kinase (PERK) branch of the UPR reduces protein translation and abundance. We hypothesized that PERK inhibition could prevent ion channel downregulation and reduce arrhythmia risk after myocardial infarct (MI). MI induced in mice by coronary artery ligation resulted in reduced ion channel levels, ventricular tachycardia (VT), and prolonged corrected intervals between the $Q$ and T waves on the ECGs (QTc). Protein levels of major cardiac ion channels were decreased. MI cardiomyocytes showed significantly prolonged action potential duration and decreased maximum upstroke velocity. Cardiac-specific PERK KO reduced electrical remodeling in response to MI, with shortened QTc intervals, fewer VT episodes, and higher survival rates. Pharmacological PERK inhibition had similar effects. In conclusion, we found that activated PERK during MI contributed to arrhythmia risk by the downregulation of select cardiac ion channels. PERK inhibition prevented these changes and reduced arrhythmia risk. These results suggest that ion channel downregulation during $\mathrm{MI}$ is a fundamental arrhythmia mechanism and that maintenance of ion channel levels is antiarrhythmic.
\end{abstract}

\section{Introduction}

Human ischemic cardiomyopathy is associated with an increased risk of sudden death and changes in multiple cardiac ion channels and transporters (1-4). The cardiac $\mathrm{Na}^{+}$channel $\left(\mathrm{Na}_{\mathrm{v}} 1.5\right)$ governs action potential (AP) phase 0 depolarization. Cardiac $\mathrm{K}^{+}$and $\mathrm{Ca}^{2+}$ channels determine the characteristic plateau phases 2 and 3 of the AP. Inward rectifying $\mathrm{K}^{+}$channels set the resting membrane potential. Reductions in these ion channels can lead to electrical remodeling and arrhythmias.

Adult cardiomyocytes lack significant regenerative potential and require a vital balance of their contents, such as sarcomeres, membrane ion channel proteins, and mitochondria to maintain viability and function throughout the life of the individual. Therefore, protein quality control is crucial for cardiomyocyte survival and function. The unfolded protein response (UPR) is one of the important mechanisms of protein quality control in the ER to monitor and regulate misfolded and unfolded proteins (5). Stimuli such as oxidative stress, hypoxia, and ischemic insult can induce ER stress and trigger the UPR. These stresses trigger complicated signaling cascades of the 3 UPR branches: dsRNA-activated protein kinase-like ER kinase (PERK), inositol-requiring ER-tonucleus signal kinase 1 (IRE1), and activating transcription factor $6 \alpha(\operatorname{ATF} 6 \alpha)$. The downstream effectors for the 3 UPR branches are

Conflict of interest: The authors have declared that no conflict of interest exists. Copyright: @ 2021, American Society for Clinical Investigation.

Submitted: January 19, 2021; Accepted: July 28, 2021; Published: September 15, 2021. Reference information: J Clin Invest. 2021;131(18):e147836.

https://doi.org/10.1172/JCl147836. phosphorylated PERK (p-PERK), phosphorylated translation initiation factor $2 \alpha$ (p-eIF $2 \alpha$ ) and activating transcription factor 4 (ATF4) for PERK; p-IRE1 and spliced X-box binding protein 1 (sXBP1) for IRE1; and the cleaved N-terminus of ATF6 $\alpha$ (ATF6N) for ATF6 $\alpha$, respectively. The PERK and IRE1 branches inhibit protein synthesis and enhance mRNA and protein degradation.

In the past decade, the UPR has been found to play important roles, mainly in pathological cardiac hypertrophy (6-8), dilated cardiomyopathy (9), ischemia/reperfusion (10-12), diabetic heart disease $(13,14)$, cardiac arrhythmias (15-17), and human heart failure $(2,18)$. Activation of all 3 UPR branches has been reported with elevated mRNA and protein levels of p-PERK/ATF4, p-IRE1/ sXBP1, and ATF6 in ischemia/reperfusion (11, 12, 19-22). Our recent studies have shown that the PERK branch of the UPR contributes to the downregulation of cardiac ion channels in human heart failure and in human induced pluripotent stem cell-derived cardiomyocytes (hiPSC-CMs) under ER stress $(2,17)$.

Many cardiac ion channels are downregulated in cardiomyopathy. For example, the $\mathrm{Na}_{\mathrm{v}} 1.5$ current $\left(\mathrm{I}_{\mathrm{Na}}\right)$ is reduced (23). Reduced $I_{\mathrm{Na}}$ jeopardizes impulse propagation, causing conduction velocity decreases and ventricular arrhythmias (2, 8, 24-26). Prolongation of the ventricular AP duration (APD) is observed in human ischemic cardiomyopathy, and animal models have revealed reductions in $\mathrm{K}^{+}$currents including the transient outward current $\left(\mathrm{I}_{\mathrm{to}}\right)$ conducted by the rapidly inactivating $\mathrm{K}^{+}$channel $\left(\mathrm{K}_{\mathrm{v}} 4.3\right)$, the inward rectifier $\mathrm{K}^{+}$current $\left(\mathrm{I}_{\mathrm{K} 1}\right)$ conducted by the inward rectifier $\mathrm{K}^{+}$channel (Kir2.1), and the slow delayed rectifier $\mathrm{K}^{+}$current $\left(\mathrm{I}_{\mathrm{Ks}}\right)$ conducted by the slowly inactivating $\mathrm{K}^{+}$channel (K LQT1) (27-31). These current reductions have been linked to reduced transcrip- 


\section{Table 1. Characterization of MI mouse heart function, cellular parameters of AP and ion channel currents, and channel protein levels, 3 weeks after MI surgery}

Sham

MI

\begin{tabular}{|c|c|c|}
\hline \multicolumn{3}{|l|}{ Heart function } \\
\hline $\mathrm{EF} \%$ (no. of mice) & $55.3 \% \pm 1.6 \%(25)$ & $24.2 \% \pm 1.4 \%(38)^{\mathrm{A}}$ \\
\hline VT episodes & 0 of 5 mice & 6 of 7 mice $^{A}$ \\
\hline QTC & $43.2 \pm 1.2 \mathrm{~ms}$ (no. of mice) (11) & $74.9 \pm 3.5 \mathrm{~ms}(8)^{\mathrm{A}}$ \\
\hline \multicolumn{3}{|l|}{ Cell APs } \\
\hline $\mathrm{APD}_{90}$ & $100 \pm 12 \mathrm{~ms}(23)$ & $291 \pm 43 \mathrm{~ms}(19)^{A}$ \\
\hline $\mathrm{dV} / \mathrm{dt}_{\max }$ & $132 \pm 6 \mathrm{~V} / \mathrm{s}(23)$ & $95 \pm 4 \mathrm{~V} / \mathrm{s}(19)^{\mathrm{A}}$ \\
\hline
\end{tabular}

Channel currents $(\mathrm{pA} / \mathrm{pF})$

$\begin{array}{lcc}\mathrm{I}_{\text {Na }} & -36.6 \pm 2.1(36) & -21.9 \pm 1.9(33)^{\mathrm{A}} \\ \mathrm{I}_{\text {Cal }} & -5.8 \pm 0.3(15) & -3.1 \pm 0.5(25)^{\mathrm{A}} \\ \mathrm{I}_{\text {to }} & 8.3 \pm 1.2(15) & 2.2 \pm 0.4(15)^{\mathrm{A}} \\ \mathrm{I}_{\mathrm{K} 1} & -11.2 \pm 1.2(20) & -6.2 \pm 0.5(20)^{\mathrm{A}} \\ \mathrm{I}_{\text {Kur }} & 11.2 \pm 1.2(19) & 4.5 \pm 0.6(15)^{\mathrm{A}}\end{array}$

Channel protein (GAPDH as loading control, normalized to sham)

$\begin{array}{lll}\mathrm{Na}_{v} 1.5 & 1.00 \pm 0.05(7) & 0.50 \pm 0.03(7)^{A} \\ \mathrm{Ca}_{\mathrm{v}} 1.2 & 1.00 \pm 0.04(7) & 0.36 \pm 0.06(7)^{\mathrm{A}} \\ \mathrm{K}_{\mathrm{v}} 4.3 & 1.00 \pm 0.06(7) & 0.49 \pm 0.07(7)^{\mathrm{A}} \\ \mathrm{Kir}_{2} .1 & 1.00 \pm 0.07(7) & 0.33 \pm 0.08(7)^{\mathrm{A}} \\ \mathrm{K}_{\mathrm{v}} 1.5 & 1.00 \pm 0.12(7) & 0.43 \pm 0.05(7)^{A}\end{array}$

Channel mRNA (Gapdh as reference gene)

$\begin{array}{lll}\mathrm{Na}_{v} 1.5 & 0.71 \pm 0.10(3) & 0.40 \pm 0.04(5)^{A} \\ \mathrm{Ca}_{\mathrm{v}} 1.2 & 1.85 \pm 0.16(3) & 0.75 \pm 0.03(5)^{\mathrm{A}} \\ \mathrm{K}_{v} 4.3 & 0.44 \pm 0.03(3) & 0.18 \pm 0.00(5)^{\mathrm{A}} \\ \mathrm{Kir}_{2} .1 & 1.92 \pm 0.10(3) & 1.34 \pm 0.12(5)^{\mathrm{A}} \\ \mathrm{K}_{\mathrm{v}} 1.5 & 0.96 \pm 0.20(3) & 0.41 \pm 0.02(5)^{\mathrm{A}}\end{array}$

Numbers in parentheses indicate the number of mice (heart function and channel protein levels) or cardiomyocytes (cell APs and channel currents) tested for each data point. Cardiomyocytes were isolated from the free wall of the left ventricle remote from the infarct using 3-5 mice from each group. Currents were measured at different resting membrane potentials $\left(\mathrm{I}_{\mathrm{Na}}:-20 \mathrm{mV} ; \mathrm{I}_{\mathrm{Ca} \mathrm{L}}:+10 \mathrm{mV} ; \mathrm{I}_{\text {to }}\right.$ and $\left.\mathrm{I}_{\mathrm{Ku}}:+50 \mathrm{mV}, \mathrm{I}_{\mathrm{K} 1 \mathrm{r}}:-100 \mathrm{mV}\right)$ and normalized to the cell capacitance. Protein levels were normalized to the sham group. ${ }^{A} P<0.05$ versus sham-operated mice, by 2 -tailed Student's $t$ test. Channel protein levels were combined from Figure 4 and Figure 6B.

tion, translation, and expression of the corresponding channels, such as $\mathrm{K}_{\mathrm{v}} 4.3$, Kir2.1, $\mathrm{K}_{\mathrm{v}} \mathrm{LQT} 1$, as well as of accessory proteins including minK and $\mathrm{K}^{+}$channel-interacting protein $2(28,31,32)$.

Here, we tested whether PERK contributed to the ion channel reductions and arrhythmias seen after myocardial infarction (MI) and whether PERK inhibition could improve the risk for arrhythmia by preventing the reduction in ion channels seen with ischemic cardiomyopathy.

\section{Results}

Mice with induced MI have QT prolongation, arrhythmias, and suppression of ion channels. Mice subjected to induced MI (referred to hereafter as MI mice) showed significant cardiomyopathy by week 3 after MI surgery (Table 1). The ejection fraction (EF) percentage was significantly decreased to $24.2 \% \pm 1.4 \%$ (vs. $55.3 \% \pm 1.6 \%$ for sham-operated mice, $P<0.0001)$. The corrected interval between
Q and T waves (QTc) on ECG was significantly prolonged to $74.9 \pm$ $3.5 \mathrm{~ms}$ (vs. $43.2 \pm 1.2 \mathrm{~ms}$ for the sham-operated mice, $P<0.0001$ ). Telemetry showed ventricular tachycardia (VT) episodes in 6 of 7 MI mice but in none of the 5 sham-operated mice $(P<0.05)$. We observed sudden death by telemetry for 4 mice. Of these, 3 mice experienced VT, and 1 died of bradycardia.

At the cellular level, cardiomyocytes remote from the ischemic zone had a significantly prolonged APD (APD ${ }_{90}: 291 \pm 43 \mathrm{~ms}$ vs. 100 \pm 12 ms for sham-treated mice, $P<0.05)$ and a decreased maximum velocity of the AP upstroke $\left(\mathrm{dV}_{\mathrm{dt}} \mathrm{dt}_{\max }: 95 \pm 4 \mathrm{~V} / \mathrm{s}\right.$ vs. $132 \pm 6 \mathrm{~V} / \mathrm{s}$ for sham-operated mice, $P<0.05$ ) (Table 1). All cardiac ion channels were downregulated at the protein and current levels in the MI group when compared with the sham group (Table 1). The reduction in protein levels varied from a $36 \% \pm 10 \%$ reduction of the $\mathrm{K}_{v} 4.3$ channel to a $75 \% \pm 7 \%$ reduction of the $\mathrm{L}$-type $\mathrm{Ca}^{2+}$ channel $\left(\mathrm{Ca}_{\mathrm{v}} 1.2, \mathrm{P}<0.05 \mathrm{vs}\right.$. sham-operated mice for all). The $\mathrm{I}_{\mathrm{Na}}$ and L-type calcium channel current $\left(\mathrm{I}_{\mathrm{CaI}}\right)$ were decreased by $40 \% \pm 6 \%$ and $47 \% \pm 9 \%$, respectively $(P<0.001)$, and the $\mathrm{K}^{+}$currents $\left(\mathrm{I}_{\mathrm{to}}, \mathrm{I}_{\mathrm{K} 1}\right.$, and the ultrarapid delayed rectifier $\mathrm{K}^{+}$current $\left[\mathrm{I}_{\mathrm{Kur}}\right]$ conducted by the voltage-gated $\mathrm{K}^{+}$channel, shaker-subfamily, member $\left.5, \mathrm{~K}_{\mathrm{v}} 1.5\right)$ were decreased by $73 \% \pm 6 \%$, $45 \% \pm 7 \%$, and $60 \% \pm 7 \%$, respectively $(P<0.0001)$. Representative traces and the current-voltage relationship curves of the cardiac ion channels are shown in Supplemental Figure 1 (supplemental material available online with this article; https://doi.org/10.1172/ JCI147836DS1). We observed no significant difference in the gating properties of ion channels, indicating that the reduced currents mainly resulted from the decrease in channel protein expression.

The UPR was activated in MI mouse hearts. As shown in Figure 1, all 3 UPR branches were activated under MI, with elevated protein levels of p-PERK, p-IRE1, and ATF6N. Significant elevations of p-PERK were observed starting 1 week after MI.p-IRE1 and ATF6N levels took an additional week to rise. All 3 UPR branches continued to be activated at week 3 . We performed similar experiments on sham-operated mice and observed no significant increase in p-PERK, p-IRE1, or ATF6N levels (Supplemental Figure 2).

PERK inhibition improved adverse electrical remodeling after MI. Three weeks after surgery, the MI mice exhibited significantly reduced systolic function, and all 3 UPR effectors were activated. At this time point, we initiated treatment with the PERK inhibitor GSK2606414 (GSK). A 3-week treatment with GSK $(100 \mathrm{mg} / \mathrm{kg} /$ day) led to decreased protein levels of p-PERK but had no effects on IRE1 or ATF6N levels, confirming the drug's specificity (Figure 2).

Pharmacologic inhibition of PERK by GSK oral treatment significantly improved the cellular APs, channel currents, and protein expression levels. As shown in Figure 3, A and B, GSK shortened the APD (APD at $90 \%$ of repolarization $\left[\mathrm{APD}_{90}\right]: 174 \pm 17 \mathrm{~ms}$ vs. $291 \pm 43 \mathrm{~ms}$ of MI, $P<0.01)$ and increased the $\mathrm{dV} / \mathrm{dt}_{\max }(108 \pm 2 \mathrm{~V} / \mathrm{s}$ vs. $95 \pm 4 \mathrm{~V} / \mathrm{s}$ of $\mathrm{MI}, P<0.01$ ), a parameter associated with $\mathrm{Na}_{\mathrm{v}} 1.5$ channel availability and conduction velocity. GSK prevented the reduction of $\mathrm{Na}_{\mathrm{v}} 1.5$ and $\mathrm{K}_{\mathrm{v}} 4.3$ in $\mathrm{MI}$ myocytes. $\mathrm{I}_{\mathrm{Na}}$ and $\mathrm{I}_{\mathrm{to}}$ currents in the MI-GSK-treated group were increased to $-33.3 \pm 2.9$ and 5.1 $\pm 1.0 \mathrm{pA} / \mathrm{pF}$, respectively ( $P<0.001$ vs. MI mice; Figure $3 \mathrm{C}$ ). Correspondingly, the protein levels of $\mathrm{Na}_{\mathrm{v}} 1.5$ and $\mathrm{K}_{\mathrm{v}} 4.3$ were increased (Figure 4). These data indicated a PERK-dependent regulation of these 2 channels in MI hearts. GSK treatment also improved protein expression of $\mathrm{K}_{\mathrm{v}} 1.5$ in MI hearts (0.88- \pm 0.05-fold vs. sham-operated mice, $P<0.05$ vs. MI and $P>0.05$ vs. sham), 

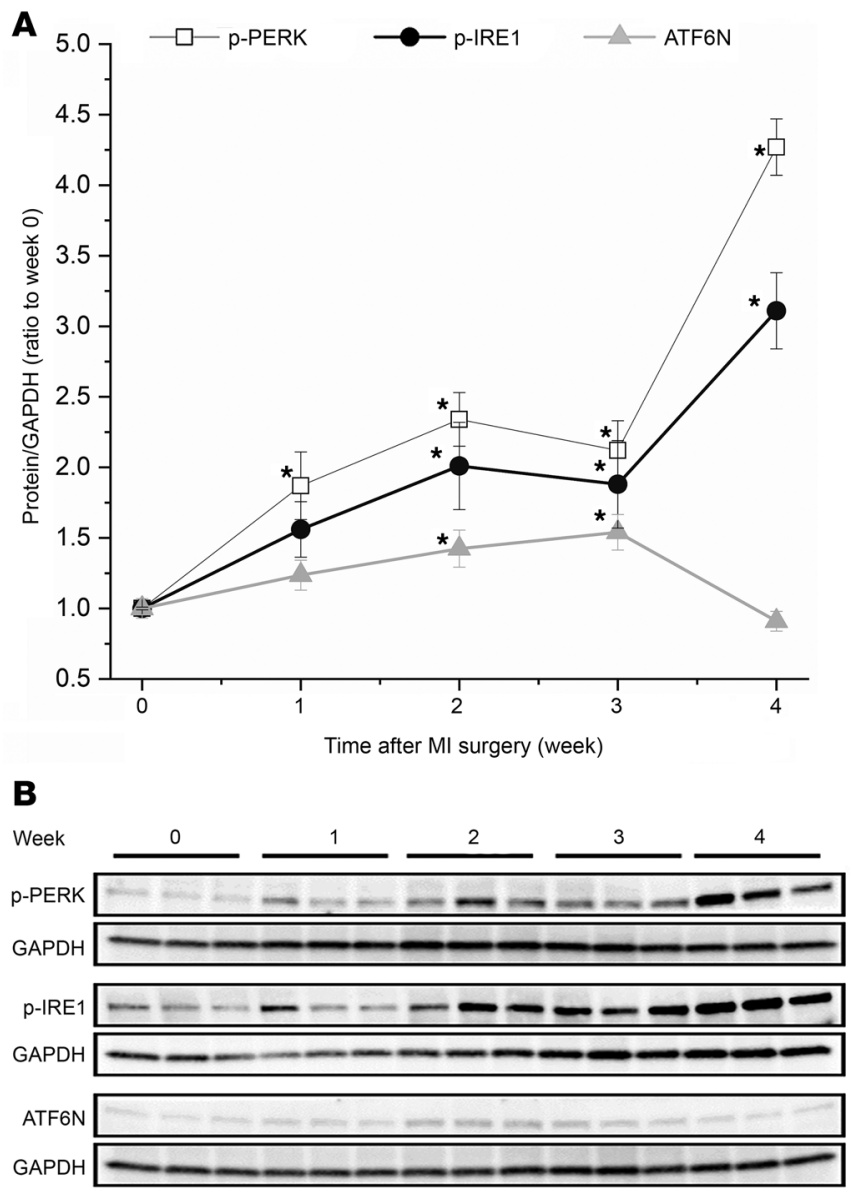

although the $\mathrm{I}_{\mathrm{Kur}}$ was not recovered in MI cardiomyocytes treated with GSK. GSK treatment was associated with reduced VT and improved survival when compared with MI alone (Supplemental Figure 3). We found, however, that PERK inhibition did not affect all channels equally. GSK treatment did not reverse the decreased current and protein levels of $\mathrm{Ca}_{\mathrm{v}} 1.2$ and Kir2.1 in MI mice, indicating that the downregulation of these channels in MI was probably PERK independent. Representative traces and the current-voltage relationship curves of the cardiac ion channels are shown in Supplemental Figure 1. GSK treatment of sham-operated mice showed unaltered APD and conduction velocity (Supplemental Figure 4).

PERK KO in MI mice. To study PERK regulation of MI hearts at the animal level, we generated constitutive, cardiac-specific PERKKO mice. PERK-KO mice showed normal heart function at baseline $(\mathrm{EF}=53.8 \% \pm 2.4 \%, P=0.67$ vs. sham-operated mice). As shown in Figure 5, three weeks after MI surgery was performed on cardiac-specific PERK-KO mice (referred to hereafter as MI-PERK-KO mice), these mice had a higher survival rate (35 of 51 MI-PERK-KO mice vs. 24 of 54 WT MI mice, $P<0.05$; see Supplemental Figure 5 for the survival curves for MI and MI-PERK-KO mice) and a somewhat smaller reduction in the $\mathrm{EF}(31.3 \% \pm 2.5 \%$ for MI-PERK-KO vs. $24.2 \% \pm 1.4 \%$ for MI mice, $P<0.01$ ). We compared the infarct scar sizes of MI (epicardial infarct ratio: $0.48 \pm 0.06$; endocardial infarct ratio: $0.60 \pm 0.03$ ) and MI-PERK-KO (epicardial infarct ratio: $0.48 \pm 0.03$; endocardial infarct ratio: $0.55 \pm 0.03)$ mouse hearts and found no significant difference (Supplemental Figure 5).
Figure 1. All 3 UPR branches were activated after MI. (A) The timeline of UPR activation in MI mouse heart tissue before (week 0 ) and after (weeks 1-4) the Ml operation shows elevated protein expression of p-PERK, p-IRE1, and ATF6N. ${ }^{*} P<0.05$ compared with week 0 , by 1-way ANOVA with post hoc tests of significance corrected for multiple comparisons. (B) Protein bands obtained by Western blotting. Three left ventricles from mice were tested for each data point.

The prolonged QTc intervals were shortened in MI-PERK-KO mice $(63.8 \pm 3.2 \mathrm{~ms})$ as compared with those for MI mice $(74.9 \pm$ $3.5 \mathrm{~ms}, P<0.05)$. As shown in Figure 5D, only 1 of 7 MI-PERK-KO mice had VT episodes, which was a significantly lower number than for the MI mice ( 6 of 7 mice, $P<0.05$ ). The benefit of PERK $\mathrm{KO}$ on the post-MI arrhythmia risk was also confirmed with optical mapping studies (Supplemental Figure 6). MI-PERK-KO mice showed significantly less arrhythmia inducibility compared with MI mice $(P<0.0001)$. This improvement in arrhythmia risk was accompanied by a reduction in APD heterogeneity, which was calculated as the difference between the APDs measured in the MI border and those in the remote zones of the left ventricle. MI mice showed significant APD heterogeneity, thus creating substrates for conduction blocks and arrhythmias. On the other hand, the MI-PERK-KO hearts had similar APDs in the entire left ventricle and significantly less heterogeneity $(P=0.0138$ vs. MI mouse hearts), which could explain the significant decrease in arrhythmia inducibility. Analysis of premature ventricular contractions was undertaken by telemetry. We analyzed 2 hours of continuous ECG recordings (1-2 am and 1-2 pm) and noted no significant difference in ventricular premature beats (VPBs) between the MI and MI-PERK-KO mice (Supplemental Figure 6). The telemetry data showed no significant difference in the heart rate when the mice were at rest $(575 \pm 15 \mathrm{bpm}$ for MI mice vs. $575 \pm 17$ bpm for MI-PERK-KO mice, respectively; data were collected from 1 to $2 \mathrm{pm}$ ).

As shown in Figure 6, the protein changes of the UPR effectors and ion channels in the MI-PERK-KO mouse hearts were similar to those seen with GSK inhibition. The significant elevation of p-PERK in MI mouse hearts was abolished in MI-PERKKO hearts. Residual PERK levels seem likely to have originated from cells other than cardiomyocytes. The protein levels of p-IRE1 and ATF6 $\mathrm{N}$ were still significantly higher in MI-PERK-KO hearts compared with levels in sham-operated mouse hearts, but no significant difference was observed when compared with levels in MI mouse hearts. For the cardiac ion channels, we obtained similar results with the PERK-KO hearts when compared with pharmacological inhibition with GSK. The marked reductions of $\mathrm{Na}_{\mathrm{v}} 1.5, \mathrm{~K}_{\mathrm{v}} 4.3$, and $\mathrm{K}_{\mathrm{v}} 1.5$ in MI hearts were reversed, whereas $\mathrm{Ca}_{\mathrm{v}} 1.2$ and Kir2.1 were still significantly decreased in MI-PERKKO hearts. MI was associated with a correlated reduction in ion channel mRNA, current, and protein levels (Supplemental Figure 1). PERK inhibition improved these values for $\mathrm{Na}_{v} 1.5, \mathrm{~K}_{v} 4.3$, and $\mathrm{K}_{\mathrm{v}} 1.5$ channels, suggesting that the ion channel reductions seen with MI were PERK dependent and that the primary effect of PERK inhibition was at the level of mRNA abundance. While it is possible that a changing complement of cell types in the heart could alter normalization results for protein in Western blots when using whole-heart lysates from the 3 experimental condi- 


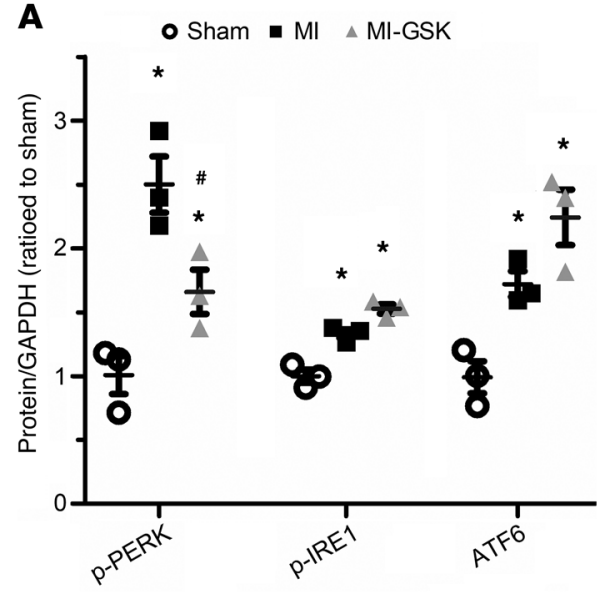

B

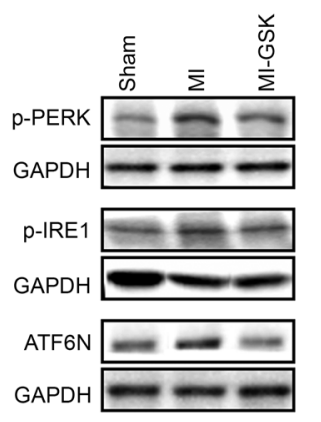

Figure 2. CSK specifically inhibited PERK activation. (A) Specific inhibition of the PERK branch of the UPR by GSK $(100 \mathrm{mg} / \mathrm{kg} /$ day for 3 weeks) was confirmed by the reduced protein levels of p-PERK in MI-CSK mouse hearts after treatment. Three mouse left ventricles were tested for each group. Three left ventricles from mice in the sham and $\mathrm{Ml}$ groups were collected 3 weeks after sham or MI surgery, respectively. Three left ventricles were collected from MI-GSK-treated mice 6 weeks after the MI surgery that was followed by 3 weeks of CSK treatment starting 3 weeks after surgery. ${ }^{*} P<0.05$ versus sham; ${ }^{\#} P<0.05$ versus MI, by 1-way ANOVA with post hoc correction. (B) Representative protein bands in Western blots. tions, a high degree of correlation between PERK-dependent ion channel current, protein, and mRNA changes strongly indicates that these are changes in myocyte biology.

\section{Discussion}

Ischemic heart disease is responsible for three-quarters of sudden cardiac deaths. Infarction induces ER stress and activates all 3 UPR branches $(10,15,19,33-35)$. In this study, we observed UPR activation with significant elevation of p-PERK, p-IRE1, and ATF6N levels in MI hearts, which was associated with downregulation of all major cardiac ion channels. The results of our inhibition studies showed that the reductions in $\mathrm{Na}_{\mathrm{v}} 1.5, \mathrm{~K}_{\mathrm{v}} 4.3$, and $\mathrm{K}_{\mathrm{v}} 1.5$ were PERK dependent. At the cellular level, pharmacologic inhibition of PERK prevented the downregulation of these channels and reduced deleterious electrical remodeling (Figure 3). Genetic inhibition of PERK at the time of MI confirmed the pharmacological results and demonstrated that PERK inhibition improves survival rates, improves the EF somewhat, shortens the QTc interval, and reduces the risk for arrhythmia after MI (Figure 5). Optical mapping experiments showed that PERK inhibition reduced arrhythmia inducibility and that the MI-PERK-KO left ventricle had significantly less heterogeneity (the difference in APD) between the MI region and the remote zone compared with MI hearts (Supplemental Figure 6). These data reinforce the basic finding that PERK inhibition reduced the post-MI arrhythmia risk and provide a mechanistic link between the electrophysiological changes and reduced arrhythmia risk. Nevertheless, it is possible that some portion of the survival benefit accrued with PERK inhibition was not related to the ion channel chang-
A

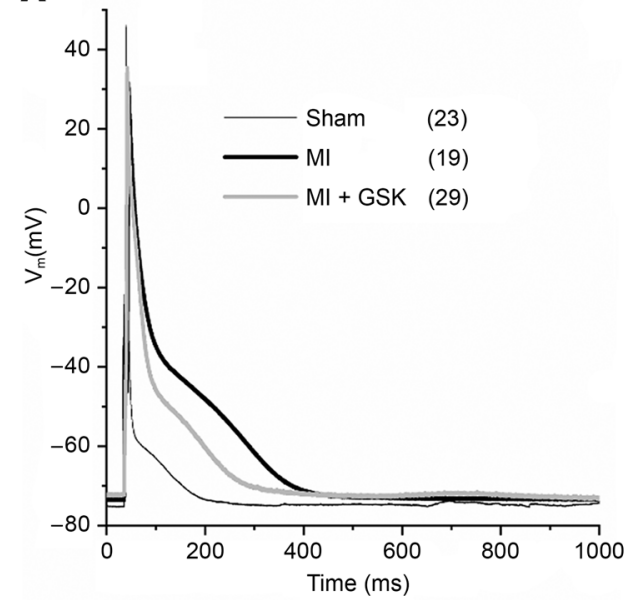

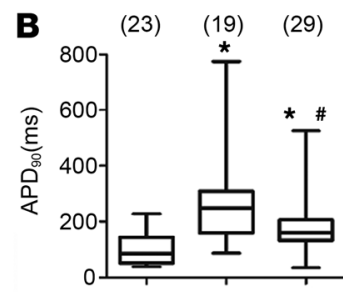

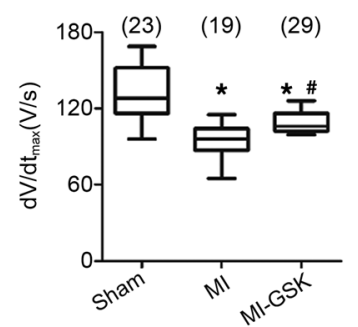

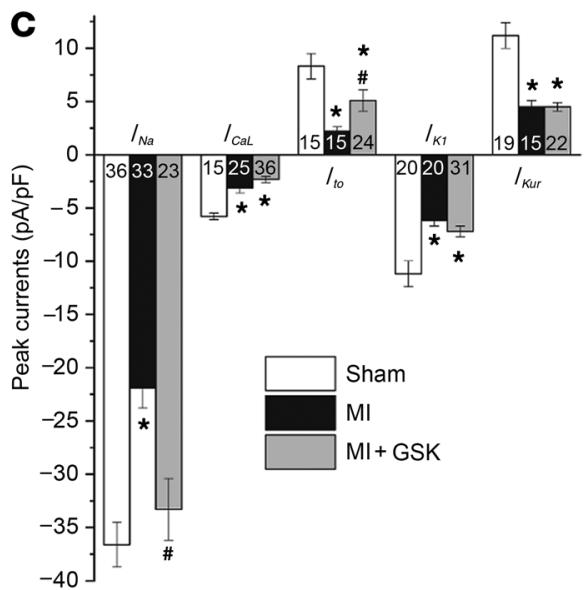

Figure 3. PERK inhibition by GSK treatment partially reversed the changes in AP and cardiac channel currents as a result of MI. (A) The prolonged APD in MI cardiomyocytes was shortened by GSK treatment (100 mg/kg/day for 3 weeks). $V_{m}$, membrane potential. (B) The prolonged APD ${ }_{90}$ and decreased $\mathrm{dV} / \mathrm{dt}_{\max }$ were partially reversed by GSK. The numbers in parentheses in $\mathbf{A}$ and $\mathbf{B}$ indicate the number of cardiomyocytes tested. (C) All major cardiac ion channel currents were decreased in MI cardiomyocytes, among which $\mathrm{I}_{\mathrm{Na}}$ and $\mathrm{I}_{\text {to }}$ were restored by GSK treatment. Fifteen to 36 cardiomyocytes (shown in parentheses or in the bars) isolated from the remote zone of 3-5 mouse left ventricles were tested for each group. Cardiomyocytes were isolated from sham and MI mice at the end of week 3 after sham or MI surgery, respectively. Cardiomyocytes from mice in the MI-CSK group were isolated 6 weeks after the $\mathrm{MI}$ surgery that was followed by followed by 3 weeks of GSK treatment starting 3 weeks after surgery. ${ }^{*} P<0.05$ versus sham; ${ }^{*}<0.05$ versus $\mathrm{MI}$, by 1-way ANOVA with post hoc correction. 
A

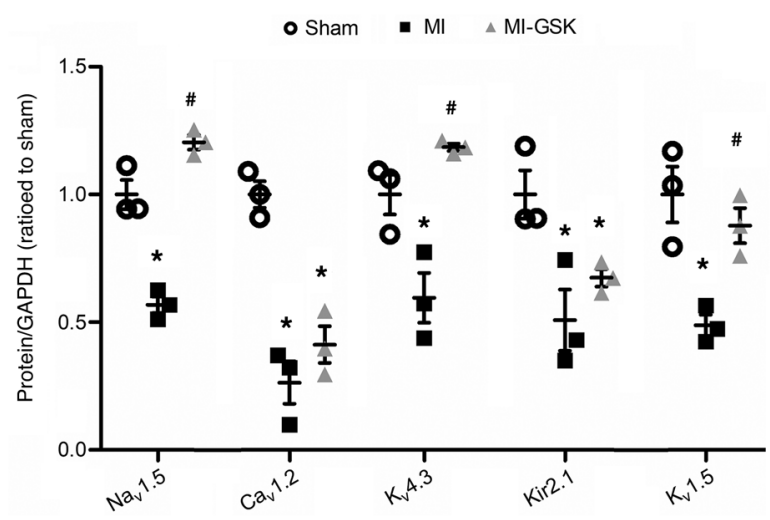

B

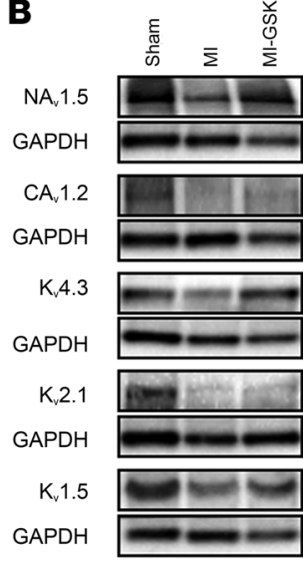

Figure 4. Pharmacologic inhibition of PERK by GSK prevented protein reductions of $\mathrm{Na}_{\mathrm{v}} 1.5, \mathrm{~K}_{\mathrm{v}} 4.3$, and $\mathrm{K}_{\mathrm{v}} 1.5$, but not $\mathrm{Ca}_{\mathrm{v}} 1.2$ or Kir2.1. (A) CSK ( $100 \mathrm{mg} / \mathrm{kg} /$ day for 3 weeks) prevented reductions of $\mathrm{Na}_{v} 1.5, \mathrm{~K}_{v} 4.3$, and $\mathrm{K}_{\mathrm{v}} 1.5$ channel proteins. (B) Representative protein bands from Western blots. Three mouse left ventricles were tested for each group. All tissue samples were taken from comparable locations of the left ventricle free wall. Left ventricles were isolated from mice in the sham and $\mathrm{Ml}$ groups at the end of week 3 after sham or Ml surgery, respectively. Left ventricles were isolated from mice in the MI-CSK group 6 weeks after the MI surgery that was followed by 3 weeks of GSK treatment starting 3 weeks after surgery. ${ }^{*} P<0.05$ versus sham mice; ${ }^{\#} P$ $<0.05$ versus $\mathrm{MI}$ mice, by 1 -way ANOVA with post hoc correction. es observed or to a reduction in arrhythmia risk. On the other hand, improved survival in the absence of significant changes in infarct size or EF strongly suggests that the major treatment effect was on arrhythmia risk. The scheme in Figure 7 summarizes the effects of UPR inhibition on MI-induced ion channel downregulation and increased arrhythmia risk.

In both humans and mice, sudden death can occur from bradyor tachyarrhythmias. Our telemetry results confirmed a preponderance of tachycardia prior to death. Moreover, PERK inhibition improved established markers of arrhythmia risk including partial reversal of ion channel electrical remodeling, shortened APDs and QTc intervals, reduced VT episodes, improved AP heterogeneity, and reduced ventricular arrhythmia inducibility. Paradoxically, VPB frequency, a predictor of human sudden death, was unchanged by PERK inhibition (Supplemental Figure 5). VPB frequency was previously found in mice to be a poor arrhythmia risk predictor (36), and VPB frequency may have different predictive implications between species. Despite any species differences in
A

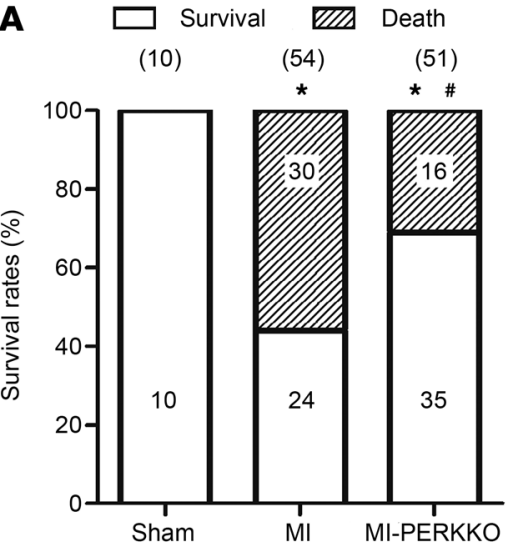

B

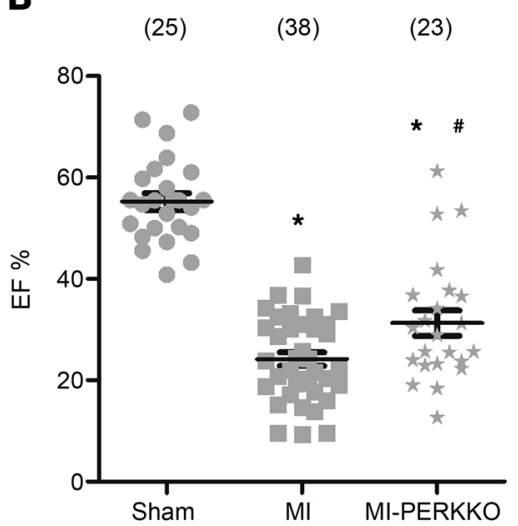

Figure 5. Genetic inhibition of PERK improved survival, EF percentage, QTC intervals, and VT after MI. (A) PERK KO in MI improved MI survival rates. (B) PERK KO in MI slightly improved the EF percentage. (C) PERK KO in MI reduced QTc interval prolongation. (D) PERK KO in MI reduced VT episodes. For the survival data, we monitored the mice for 4 weeks after Ml surgery. The numbers of mice tested are shown in parentheses or in the bars in A-D. All data except for the survival rates were collected at the end of week 3 after sham or $\mathrm{MI}$ surgery. ${ }^{*} P<0.05$ versus sham; ${ }^{\#} P<0.05$ versus MI, by Fisher's exact test (A and $\mathbf{D})$ and 1-way ANOVA with post correction (B and $\mathbf{C})$.

\section{C}

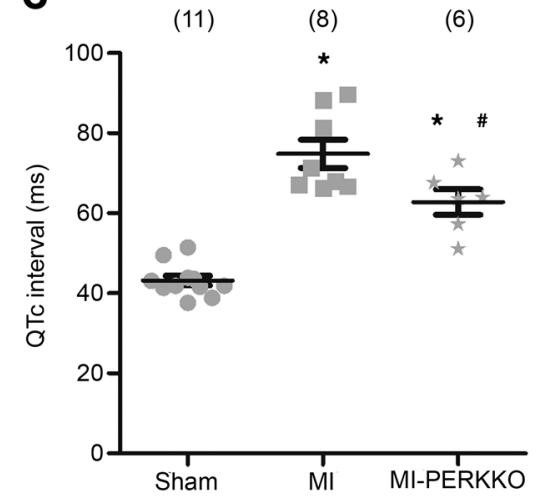

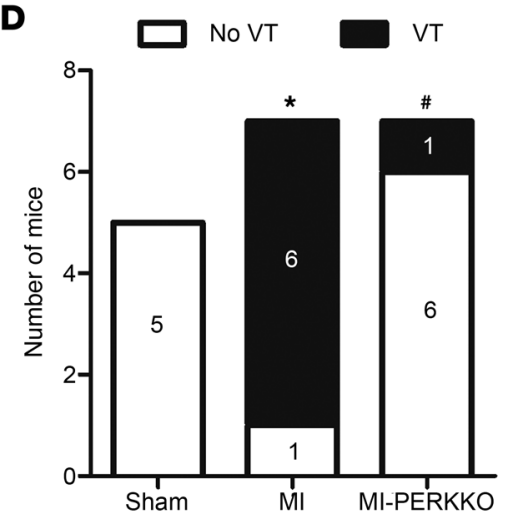


A

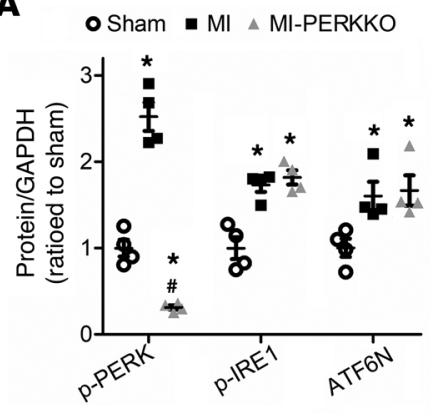

B

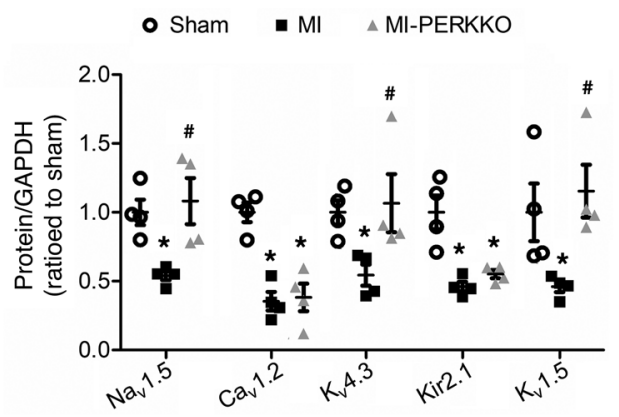

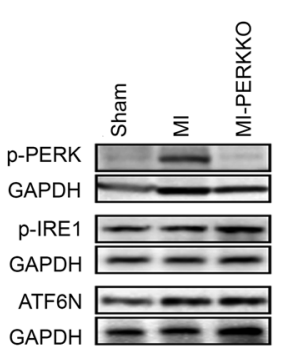
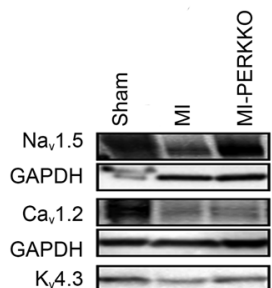

GAPDH

$\mathrm{K} 2$

GAPDH

$\mathrm{K}_{\mathrm{v}} 1.5$

GAPDH
Figure 6. PERK KO had effects on electrical remodeling similar to those seen with pharmacological inhibition. Protein levels of $(\mathbf{A})$ activated UPR effectors and (B) cardiac ion channels were altered in WT $\mathrm{MI}$ and MI-PERK-KO mouse hearts, as measured by Western blotting. Four mouse left ventricles were tested for each group. The left ventricles were collected from mice at the end of week 3 after sham or MI surgery. ${ }^{*} P<0.05$ versus sham; ${ }^{\#} P<0.05$ versus MI, by 1 -way ANOVA with post hoc correction.

the reduction in ion channels is arrhythmogenic in nature. This is consistent with genetic syndromes in which ion channels are reduced. Second, it seems reasonable to assume that some of the proarrhythmia risk of current ion channel-blocking antiarrhythmic drugs is the result of the combination of inherent ion channel reductions in response to injury and subsequent further pharmacological blockade. If this is true, preventing the injury-induced ion channel downregulation would have the additional advantage of reducing antiarrhythmic drug proarrhythmia.

In our study, PERK inhibition was antiarrhythmic and improved the survival rate but not the infarct size. PERK activation under mild ER stress can be adaptive, whereas prolonged UPR activation inhibits protein synthesis and promotes apoptosis $(15,16,33-35)$. Organ-specific and short-term inhibition of PERK has had beneficial effects in other studies (34, the mechanisms or predictors of arrhythmia death, the applicability of UPR inhibition to humans is supported by our previous findings in human heart tissue and hiPSC-CMs $(2,17)$.

Although the UPR is thought to be a general mechanism of protein control, we observed that PERK had selective effects. For example, $\mathrm{Ca}_{\mathrm{v}} 1.2$ and Kir2.1 were downregulated in MI mouse hearts with decreased protein expression and channel currents. Nevertheless, these 2 channels did not appear to be regulated by PERK, since GSK treatment or PERK KO did not reverse the protein or current changes. Another branch of the UPR, IRE1, can also inhibit protein synthesis, and the PERK-independent changes in $\mathrm{Ca}_{v} 1.2$ and Kir2.1 could be induced by the activated IRE1 in MI mouse hearts. An IRE1-dependent regulation of $\mathrm{Ca}_{v} 1.2$ has been observed in hiPSC-CMs (17).

In addition to improving electrical remodeling and arrhythmia risk when inhibited after MI, genetic PERK inhibition, but not GSK, partially protected the EF after MI. We speculate that this is because of a greater reduction in the apoptotic marker protein C/EBP homologous protein (CHOP, a PERK branch downstream) (37-39) in PERK KO with an expected, associated reduction in apoptosis (Supplemental Figure 7). These results are consistent with other studies showing that CHOP contributes to the development of heart failure and that CHOP downregulation plays protective roles against cell apoptosis $(40,41)$. Nevertheless, we observed no improvement in the EF in MI-GSK mice (Supplemental Figure 8), and no change in infarct size was noted histologically between the MI and MI-PERK-KO groups, suggesting that the main effect of PERK inhibition was not on structural remodeling. It is possible that genetic PERK inhibition may have other unidentified salutary effects not seen with GSK.

Our study showed that most ion channels were downregulated in MI and that preventing the reduction in ion channels was antiarrhythmic. These findings have several implications. First,
38, 40-42), whereas long-term PERK inhibition, especially global inhibition, may have undesirable side effects such as hyperglycemia and its downstream consequences (43-46). Until these effects are further evaluated, the experiments should be taken as proof of principle that interrupting signaling cascades that result in electrical remodeling may be beneficial. Moreover, it is possible that the increase in survival seen in this study may result in part from other effects of UPR inhibition other than just inhibition of electrical remodeling. Nevertheless, it stands to reason that reducing the QT interval would be antiarrhythmic, and preventing downregulation of cardiac $\mathrm{Na}_{\mathrm{v}} 1.5$ channels by other means during cardiomyopathy has been shown to be antiarrhythmic $(8,17,47,48)$. Therefore, it seems likely that at least some of the PERK-inhibitory effect is through electrical remodeling. PERK inhibition would be expected to have less effect with time after MI as the UPR becomes less active, and this would suggest that transient therapy may suffice to affect arrhythmias and survival after MI.

In summary, we inhibited the PERK branch of the UPR and observed reduced arrhythmia markers and risk after MI. PERK inhibition prevented selected sodium and potassium channel changes and APD prolongation. Furthermore, PERK inhibition during MI reduced the death rate and arrhythmia risk. Therefore, targeting the PERK branch of the UPR could be a novel therapeutic approach to treat ischemic heart disease.

\section{Methods}

Generation of ischemic cardiomyopathy. We used an established mouse ischemic cardiomyopathy model as described before $(8,49)$. Briefly, C57BL/6J mice (male and female mice were 11 weeks old and weighed 22-30 g; The Jackson Laboratory) were anesthetized with 1\%-3\% isoflurane in an induction chamber. Buprenorphine $(0.05-0.1 \mathrm{mg} / \mathrm{kg})$ was injected subcutaneously at the time of surgery. MI was induced 


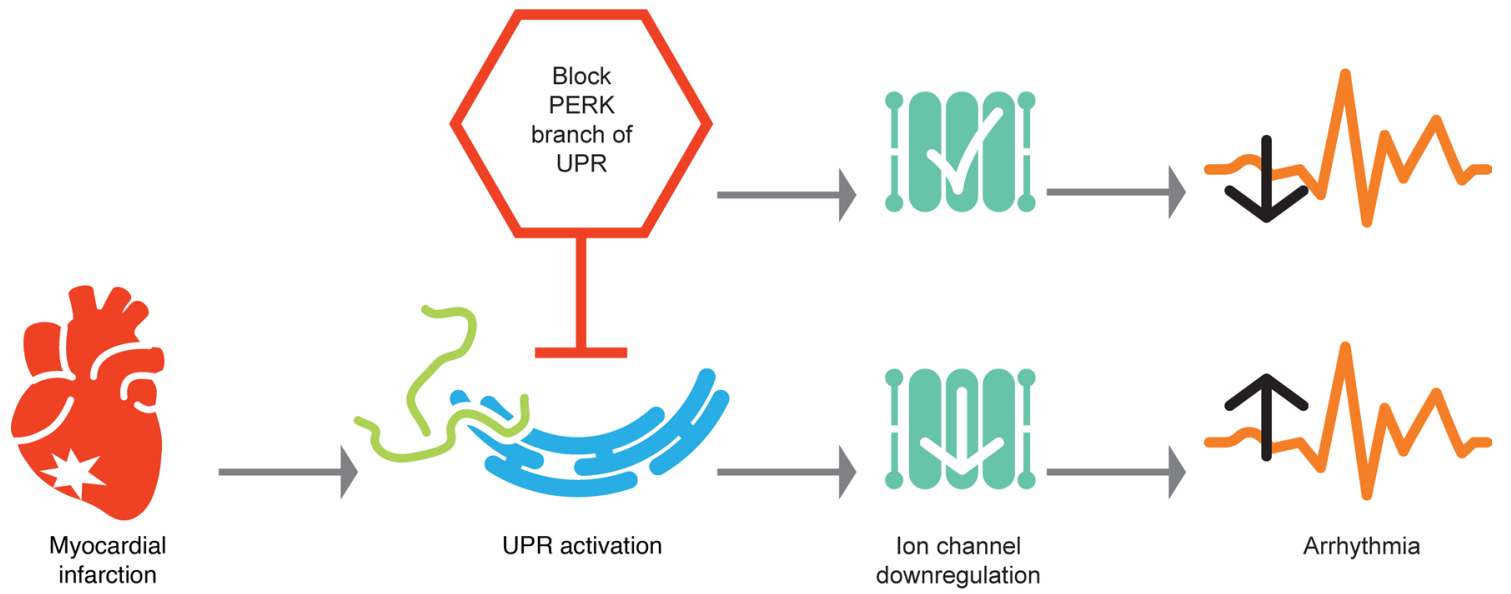

Figure 7. Scheme of the effects of UPR inhibition on MI-induced ion channel downregulation and increased arrhythmia risk. Myocardial infarction activates the UPR, which leads to the downregulation of cardiac ion channels and arrhythmias. With blockade of the PERK branch of the UPR, cardiac ion channel WT levels can be restored, and the arrhythmias from MI can be reduced.

by ligation of the left coronary artery descending branch with an 8-0 polypropylene ligature around the proximal portion of the artery in the mice. Mice that underwent sham surgeries served as controls.

PERK inhibition. Pharmacologic PERK inhibition was achieved with GSK (MedKoo Biosciences). GSK was dissolved in $0.5 \%$ hydroxypropylmethyl cellulose and $0.1 \%$ Tween- 80 and was gavage fed to mice at $100 \mathrm{mg} / \mathrm{kg} /$ day for 3 weeks. GSK alone was nontoxic to the control animals as shown in Supplemental Figure 4. Genetic inhibition was achieved by a cardiac-specific PERK KO. The C57BL/6J strain Eif2ak3 $3^{\text {tm1.2Drc }} / \mathrm{J}$ mice (The Jackson Laboratory, stock no. 023066) are floxed mutant mice possessing loxP sites flanking exons 7-9 of the Eif2ak3 gene (43). These mice were bred with B6N.FVB(B6)-Tg(Myh6-Cre)2182Mds/J mice that expressed Cre recombinase under the control of the $\alpha \mathrm{MHC}$ promoter (The Jackson Laboratory, stock no. 018972). Genotyping for Eif2ak3 and Cre was performed to confirm the cardiac-specific KO of PERK by following The Jackson Laboratory protocols 23633 and 26338, indicated on their website and shown in Supplemental Figure 9.

Animal groups. Four groups of C57BL/6J mice were studied: (a) sham-operated mice; (b) MI mice; (c) MI-GSK mice; and (d) MIPERK-KO mice. We tested the timeline of the UPR effectors in sham and MI mice before the operation (as week 0 ) and at the end of weeks 1, 2, 3, and 4 after the operation in Figure 1 and Supplemental Figure 2. For all other experiments, sham, MI, and MI-PERK-KO mice were studied 3 weeks after the operation. MI-GSK mice were started on GSK treatment 3 weeks after the MI and studied after another 3 weeks of the therapy. All mice were age matched, and mice of both sexes were used.

Echocardiography. Echocardiography was performed as in previous studies (50-52). Mice were anesthetized with $1 \%$ isoflurane via nose cone at $0.5 \mathrm{~L} / \mathrm{min}$. The ECG, respiration rate, and rectal temperature were continuously monitored with an integrated physiology platform at $37^{\circ} \mathrm{C}$. The Vevo 2100 (VisualSonics) ultrasound unit was used to record $\mathrm{M}$-mode images in the parasternal long axis and the left ventricle short-axis views at the mid-papillary level for the measurement of LV end-systolic dimensions (LVESd), LV end-diastolic dimensions (LVEDd), and LV posterior wall thickness. Measurements were averaged from 3 consecutive beats during expiration. The LV ejection fraction percentage $(\mathrm{EF} \%)$ was calculated as:

$\mathrm{EF} \%=100 \times\left[(7 / 2.4+\right.$ LVEDd $) \times$ LVEDd $\left.^{3}\right]-[(7 / 2.4+$ LVESd $) \times$ LVESd $\left.^{3}\right] /\left[(7 / 2.4+\right.$ LVEDd $) \times$ LVEDd $\left.^{3}\right]$.

Telemetry. As we have done before $(49,53)$, mice were implanted with ETA-F10 telemetry monitors (Data Sciences International) under the anesthesia of isoflurane. The 2 leads were placed in the area of the right upper chest and the left lower chest. Continuous ECG signals between 1 and 2 am and between 1 and 2 pm were recorded and evaluated using Dataquest ART (Data Sciences International). Any run of 4 or more consecutive premature ventricular contractions was defined as VT.

Histological evaluation of the infarct size in MI mouse hearts. Hearts were excised under $2 \%$ isoflurane anesthesia. $\mathrm{KCl}(10 \%)$ was injected into the $\mathrm{LV}$ chamber before the hearts were harvested to arrest them in diastole (54). The left and right atria and large vessels were resected, and the heart was washed with ice-cold PBS and fixed in $10 \%$ formalin overnight. Then, heart tissues were embedded in paraffin using a Tissue-TeK VIP processor (Sakura Finetek USA). Tissue blocks were cut at $5 \mu \mathrm{m}$ thickness with $100 \mu \mathrm{m}$ between each section through the block. Sections were stained with routine Masson's trichrome (Rowley Biochemical) according to the manufacturer's instructions. The images were taken with a NIKON Eclips E800M microscope (Nikon Instruments), and NIS-Elements D 5.02.00 64 bit imaging software (Nikon Instruments) was used. The infarct scar size was analyzed with ImageJ Fiji software (NIH). The scar size was evaluated by the endocardial and epicardial infarct ratio technique (54). The endocardial infarct ratio was obtained by dividing the sum of endocardial infarct lengths from all sections by the sum of endocardial circumferences from all sections. The epicardial infarct ratio was obtained by dividing the sum of epicardial infarct lengths from all sections by the sum of epicardial circumferences from all sections.

Cardiomyocyte isolation. Mouse cardiomyocytes were isolated from the remote zone of the left ventricle scar using a modified enzymatic digestion protocol, as we have done before $(8,55,56)$. Isolated cardiomyocytes were used for measurements of channel currents and APs.

Electrophysiology. Channel currents were measured using the whole-cell patch-clamp technique in voltage-clamp mode at room temperature, as we have done previously $(8,57,58)$. When measur- 
ing $\mathrm{K}^{+}$currents, nifedipine $(10 \mu \mathrm{M})$ was applied to block the L-type $\mathrm{Ca}^{2+}$ channel $\left(\mathrm{Ca}_{\mathrm{v}} 1.2\right)$ current $\left(\mathrm{I}_{\mathrm{CaL}}\right)$ (59). Standard pulse protocols were used to determine the current-voltage, steady-state availability and other gating behaviors $(8,59-61)$. APs were recorded under current-clamp mode with pulses of $3 \mathrm{~ms}$ in duration, 1.2-fold above the threshold intensity at a stimulation rate of $0.5-1 \mathrm{~Hz}$. The $\mathrm{APD}_{90}$ was used for comparison. All electrophysiological measurements were carried out with an Axopatch $200 \mathrm{~B}$ amplifier and an Axon Digitata 1320A A/D converter using software pCLAMP 9.2 software (all from Molecular Devices). Analog and P/4 methods were used for leak and capacity transient cancellation. All records were filtered with cutoff frequencies designed to avoid aliasing and were digitized at speeds at least 5 times the filter cutoff frequency, generally $2 \mathrm{kHz}$ and 10 $\mathrm{kHz}$, respectively. Efforts were made to use electrodes with low series resistances and to choose cells that minimize space clamp issues. Series resistance was partially compensated by feedback circuitry. Data were analyzed with Clampfit (Molecular Devices). Peak currents $(\mathrm{pA} / \mathrm{pF})$ were obtained with peak current density $(\mathrm{pA})$ normalized to cell capacitance $(\mathrm{pF})$.

Western blot analysis of proteins. Protein levels of ion channels and the UPR effectors were measured from the whole left ventricle by standard Western blotting. The antibodies used include p-PERK (Ser713) antibody (BioLegend, catalog 6494), p-IRE1 (Ser724) antibody (Thermo Fisher Scientific, catalog PA1-16927), ATF6 antibody (Proteintech, catalog 24169-1-AP), CHOP antibody (Cell Signaling Technology, catalog 2895), and GAPDH antibody (Abcam, catalog ab9484). The antibodies for ion channels were purchased from Alomone Labs, including anti-Nav1.5 (ASC-005), anti-Kv4.3 (APC-017), anti-Cav1.2 (ACC-003), and anti-Kv1.5 (APC-003), anti-Kir2.1 (APC026). HRP-conjugated goat anti-rabbit (Bio-Rad, catalog 1706515) or anti-mouse (Bio-Rad, catalog 1706516) secondary antibodies were used at a dilution of 1:5000. GAPDH was used as a loading control. Loading controls were not altered by the MI surgery as confirmed by comparison with total protein levels. All data were obtained by normalizing the target's Western blot bands with the corresponding loading control. For the timeline changes of UPR effectors before and after the sham and MI surgeries in Figure 1 and Supplemental Figure 2, the data were further normalized to the levels at week 0 .

Statistics. Values are presented as the mean \pm SEM. The 2-tailed Student's $t$ test and 1-way ANOVA with post hoc tests of significance corrected for multiple comparisons were used where appropriate. The survival data, VT episodes comparisons, and optical mapping data of arrhythmia inducibility were analyzed with the Fisher's exact test. A $P$ value of less than 0.05 was considered statistically significant.

Study approval. Animal care and interventions were undertaken in accordance with the NIH guidelines for the care and use of experimental animals, and the animal use protocol (IACUC-2002-37885A) was approved by the IACUC of the University of Minnesota.

\section{Author contributions}

ML designed research studies, conducted experiments, acquired and analyzed data, and wrote the manuscript. HL and PP conducted experiments and acquired and analyzed data. GJK, GS, FF, $\mathrm{AZ}$, and LG conducted experiments. CK analyzed data. EGT conducted experiments, analyzed data, and reviewed the manuscript. SCD designed research studies and wrote the manuscript.

\section{Acknowledgments}

This work was supported by the NIH (R01 HL104025, to SCD) and the Rhode Island Foundation (20154145, to ML).

Address correspondence to: Samuel C. Dudley Jr., Fred C. and Katherine B. Andersen Chair - Adult Cardiology, Division of Cardiology, Director of the Lillehei Heart Institute, VCRC 286 - MMC 508, 425 Delaware St., SE, Minneapolis, Minnesota 55455, USA. Email:sdudley@umn.edu.
1. Zicha S, et al. Post-transcriptional alterations in the expression of cardiac $\mathrm{Na}+$ channel subunits in chronic heart failure. J Mol Cell Cardiol. 2004;37(1):91-100.

2. Gao G, et al. Unfolded protein response regulates cardiac sodium current in systolic human heart failure. Circ Arrhythm Electrophysiol. 2013;6(5):1018-1024.

3. Kaab S, et al. Molecular basis of transient outward potassium current downregulation in human heart failure: a decrease in Kv4.3 mRNA correlates with a reduction in current density. Circulation. 1998;98(14):1383-1393.

4. Tsuji Y, et al. Potassium channel subunit remodeling in rabbits exposed to long-term bradycardia or tachycardia. Circulation. 2006;113(3):345-355.

5. Arrieta A, et al. ER protein quality control and the unfolded protein response in the heart. Curr Top Microbiol Immunol. 2018;414:193-213.

6. Ni L, et al. $\beta$-AR blockers suppresses ER stress in cardiac hypertrophy and heart failure. PLoS One. 2011;6(11):e27294.

7. Luo T, et al. 4-PBA prevents pressure overloadinduced myocardial hypertrophy and interstitial fibrosis by attenuating endoplasmic reticulum stress. Chem Biol Interact. 2015;242:99106:S0009-2797(15)30078-8.
8. Liu M, et al. Mitochondrial dysfunction causing cardiac sodium channel downregulation in cardiomyopathy. J Mol Cell Cardiol. 2013;54:2534:S0022-2828(12)00393-8.

9. Ortega A, et al. Endoplasmic reticulum stress induces different molecular structural alterations in human dilated and ischemic cardiomyopathy. PLoS One. 2014;9(9):e107635.

10. Thuerauf DJ, et al. Sarco/endoplasmic reticulum calcium ATPase-2 expression is regulated by ATF6 during the endoplasmic reticulum stress sesponse: intracellular signaling of calcium stress in a cardiac myocyte model system. J Biol Chem. 2001;276(51):48309-48317.

11. Thuerauf DJ, et al. Activation of the unfolded protein response in infarcted mouse heart and hypoxic cultured cardiac myocytes. Circ Res. 2006;99(3):275-282.

12. Jin J-K, et al. ATF6 decreases myocardial ischemia/reperfusion damage and links ER stress and oxidative stress signaling pathways in the heart. Circ Res. 2017;120(5):862-875.

13. Guo R, et al. SIRT1 suppresses cardiomyocyte apoptosis in diabetic cardiomyopathy: An insight into endoplasmic reticulum stress response mechanism. Int J Cardiol. 2015;191:36-45.

14. Lakshmanan AP, et al. The hyperglycemia stimu- lated myocardial endoplasmic reticulum (ER) stress contributes to diabetic cardiomyopathy in the transgenic non-obese type 2 diabetic rats: a differential role of unfolded protein response (UPR) signaling proteins. Int J Biochem Cell Biol. 2013;45(2):438-447.

15. Liu M, et al. Role for the unfolded protein response in heart disease and cardiac arrhythmias. Int J Mol Sci. 2016;17(1):52.

16. Liu M, Dudley SC Jr. The role of the unfolded protein response in arrhythmias. Channels (Austin). 2018;12(1):335-345.

17. Liu M, et al. Activation of the unfolded protein response downregulates cardiac ion channels in human induced pluripotent stem cell-derived cardiomyocytes. JMol Cell Cardiol. 2018;117:62-71.

18. Jensen BC, et al. Upregulation of autophagy genes and the unfolded protein response in human heart failure. Int J Clin Exp Med. 2017;10(1):1051-1058.

19. Mitra A, et al. Role of $\alpha$-crystallin B as a regulatory switch in modulating cardiomyocyte apoptosis by mitochondria or endoplasmic reticulum during cardiac hypertrophy and myocardial infarction. Cell Death Dis. 2013;4(4):e582.

20. Zhang C, et al. Unfolded protein response plays a critical role in heart damage after myocardial ischemia/reperfusion in rats. PLoS One. 
2017;12(6):e0179042.

21. Jian L, et al. Chemical chaperone 4-phenylbutyric acid reduces cardiac ischemia/reperfusion injury by alleviating endoplasmic reticulum stress and oxidative stress. Med Sci Monit. 2016;22:5218-5227.

22. Zhang W, et al. Schisandrin B ameliorates myocardial ischemia/reperfusion injury through attenuation of endoplasmic reticulum stress-induced apoptosis. Inflammation. 2017;40(6):1903-1911.

23. Liu M, et al. Cardiac sodium channnel mutations: why so many phenotypes? Nat Rev Cardiol. 2014;11(10):607-615.

24. $\mathrm{Pu}$ J, Boyden PA. Alterations of $\mathrm{Na}^{+}$currents in myocytes from epicardial border zone of the infarcted heart. A possible ionic mechanism for reduced excitability and postrepolarization refractoriness. Circ Res. 1997;81(1):110-119.

25. Valdivia $C R$, et al. Increased late sodium current in myocytes from a canine heart failure model and from failing human heart. J Mol Cell Cardiol. 2005;38(3):475-483.

26. Ufret-Vincenty CA, et al. Role of sodium channel deglycosylation in the genesis of cardiac arrhythmias in heart failure. J Biol Chem. 2001;276(30):28197-28203.

27. Sah $\mathrm{R}$, et al. Regulation of cardiac excitation-contraction coupling by action potential repolarization: role of the transient outward potassium current $\left({ }_{\mathrm{It}}\right.$ ). J Physiol. 2003;546(Pt 1):5-18.

28. Scholz EP, et al. Central role of PKC $\alpha$ in isoenzyme-selective regulation of cardiac transient outward current Ito and Kv4.3 channels. J Mol Cell Cardiol. 2011;51(5):722-729.

29. Janse MJ. Electrophysiological changes in heart failure and their relationship to arrhythmogenesis. Cardiovasc Res. 2004;61(2):208-217.

30. Li GR, et al. Transmural action potential and ionic current remodeling in ventricles of failing canine hearts. Am J Physiol Heart Circ Physiol. 2002;283(3):H1O31-H1041.

31. Nuss HB, et al. Cellular basis of ventricular arrhythmias and abnormal automaticity in heart failure. Am J Physiol. 1999;277(1 pt 2):H80-H91.

32. Rosati B, McKinnon D. Regulation of ion channel expression. Circ Res. 2004;94(7):874-883.

33. Szegezdi E, et al. ER stress contributes to ischemia-induced cardiomyocyte apoptosis. Biochem Biophys Res Commun. 2006;349(4):1406-1411.

34. Luo T, et al. Attenuation of ER stress prevents post-infarction-induced cardiac rupture and remodeling by modulating both cardiac apoptosis and fibrosis. Chem Biol Interact. 2015;225:9098:S0009-2797(14)00330-5.

35. Liu M, et al. Targeting the unfolded protein response in heart diseases. Expert Opin Ther Targets. 2014;18(7):719-723.

36. Betsuyaku T, et al. Spontaneous and inducible ventricular arrhythmias after myocardial infarction in mice. Cardiovasc Pathol. 2004;13(3):156-164.

37. Isodono $\mathrm{K}$, et al. PARM-1 is an endoplasmic reticulum molecue involved in endoplasmic reticulum stress-induced apoptosis in rat cardiac myocytes. PLoS One. 2010;5(3):e9746.

38. Ohoka N, et al. TRB3, a novel ER stress-inducible gene, is induced via ATF4-CHOP pathway and is involved in cell death. ЕMBO J. 2005;24(6):1243-1255.

39. Zinszner $\mathrm{H}$, et al. CHOP is implicated in programmed cell death in response to impaired function of the endoplasmic reticulum. Genes Dev. 1998;12(7):982-995.

40. Fu HY, et al. Ablation of C/EBP homologous protein attenuates endoplasmic reticulum-mediated apoptosis and cardiac dysfunction induced by pressure overload. Circulation. 2010;122(4):361-369.

41. Nam DH, et al. CHOP deficiency prevents methylglyoxal-induced myocyte apoptosis and cardiac dysfunction. J Mol Cell Cardiol. 2015;85:168177:S0022-2828(15)00168-6.

42. Nickson P, et al. PUMA is critical for neonatal cardiomyocyte apoptosis induced by endoplasmic reticulum stress. Cardiovasc Res. 2007;73(1):48-56.

43. Zhang $P$, et al. The PERK eukaryotic initiation factor $2 \alpha$ kinase is required for the development of the skeletal systme, postnatal growth and the function and viability of the pancreas. Mol Cell Biol. 2002;22(11):3864-3874.

44. Gupta S, et al. PERK regulates the proliferation and development of insulin-secreting beta-cell tumors in the endocrine pancreas of mice. PLOS One. 2009;4(11):e8008.

45. Li Y, et al. PERK eIF2alpha kinase regulates neonatal growth by controlling the expression of circulating insulin-like growth factor-I derived from the liver. Endocrinology. 2003;144(8):3505-3513.

46. Cornejo V, et al. Role of the unfolded protein response in organ physiology: lessons from mouse models. IUBMB Life. 2013;65(12):962-975.

47. Zhou A, et al. HuR-mediated SCN5A messenger RNA stability reduces arrhythmic risk in heart failure. Heart Rhythm. 2018;15(7):1072-1080.
48. Zhou A, et al. RNA binding protein, HuR, regulates $S C N 5 A$ expression through stabilizing MEF2C transcription factor mRNA. J Am Heart Assoc. 2018;7(9):e007802.

49. Rutledge CA, et al. c-Src kinase inhibition reduces arrhythmia inducibility and connexin 43 dysregulation after myocardial infarction. J Am Coll Cardiol. 2014;63(9):928-934.

50. Xiao HD, et al. Mice with cardiac-restricted angiotensin-converting enzyme (ACE) have atrial enlargement, cardiac arrhythmia, and sudden death. Am J Pathol. 2004;165(3):1019-1032.

51. Simpson D, et al. A tissue engineering approach to progenitor cell delivery results in significant cell engraftment and improved myocardial remodeling. Stem Cells. 2007;25(9):2350-2357.

52. Silberman GA, et al. Uncoupled cardiac nitric oxide synthase mediates diastolic dysfunction. Circulation. 2010;121(4):519-528.

53. Sovari AA, et al. Mitochondria oxidative stress, connexin 43 remodeling, and sudden arrhythmic death. Circ Arrhythm Electrophysiol. 2013;6(3):623-631.

54. Takagawa J, et al. Myocardial infarct size measurement in the mouse chronic infarction model: comparison of area- and length-based approaches. J Appl Physiol (1985). 2007;102(6):2104-2111.

55. Dias FAL, et al. The effect of myosin regulatory light chain phosphorylation on the frequencydependent regulation of cardiac function. $J \mathrm{Mol}$ Cell Cardiol. 2006;41(2):330-339.

56. Sheehan KA, Blatter LA. Regulation of junctional and non-junctional sarcoplasmic reticulum calcium release in excitation-contraction coupling in cat atrial myocytes. JPhysiol. 2003;546(1):119-135.

57. Liu M, et al. Cardiac $\mathrm{Na}^{+}$current regulation by pyridine nucleotides. Circ Res. 2009;105(8):737-745.

58. Liu M, et al. Role of protein kinase $\mathrm{C}$ in metabolic regulation of the cardiac $\mathrm{Na}^{+}$channel. Heart Rhythm. 2017;14(3):440-447.

59. Brunner $\mathrm{M}$, et al. Mechanisms of cardiac arrhythmias and sudden death in transfenic rabbits with long QT syndrome. JClin Invest. 2008;118(6):2246-2259.

60. El Gebeily G, Fiset C. 4-Hydroxytamoxifen inhibits $\mathrm{K}^{+}$currents in mouse ventricular myocytes. Eur J Pharmacol. 2010;629(1-3):96-103.

61. Liu GX, et al. Differential conditions for early after-depolarizations and triggered activity in cardiomyocytes derived from transgenic LQT1 and LQT2 rabbits. J Physiol. 2012;590(5):1171-1180. 